Frederico José Amédé́ Péret

Victor Hugo Melo ${ }^{2}$

Liv BRAGa de PaUla ${ }^{3}$

Beatriz Améla Monteiro de Andrade ${ }^{3}$

JORGE ANDRADE PINTO ${ }^{2}$

Artigos originais

Palavras-chaves

Infecções por HIV/transmissão Infecção puerperal/epidemiologia

Período pós-parto

Cesárea

Endometrite

Estudos comparativos

Keywords

HIV infections/trasnmission Puerperal infection/epidemiology

Postpartum period

Cesarean section

Endometritis

Comparative studies

Correspondêncio:

Frederico José Amedeé Péret

Rua Sergipe, 330, apto. 601 - Bairro Funcionários

CEP 30130-170 - Belo Horizonte/MG

E-mail: fredperet@ig.com.br

Recebido

$28 / 11 / 2005$

Aceito com modificacọoes

$29 / 03 / 2007$

\section{Morbidade puerperal em portadoras e não-portadoras do vírus da imunodeficiência humana}

\author{
Puerperal morbidity in HIV-infected and non-infected women
}

\section{Resumo}

OBJETIVO: comparar a morbidade de puérperas portadoras e não-portadoras do vírus da imunodeficiência humana (HIV). MÉTODOS: estudo prospectivo, controlado, realizado entre julho de 2001 e setembro de 2003, com inclusão na ocasião do parto de pacientes portadoras e não-portadoras do HIV. A morbidade foi dividida em menor (sangramento pós-parto aumentado, febre e endometrite) e maior (hemotransfusão, alterações profundas da ferida operatória e necessidade de intervenção cirúrgica), e foi avaliada quanto à presença ou não de infecção pelo HIV e o tipo de parto. Foram avaliadas 205 puérperas: 82 portadoras do HIV (grupo HIV-casos) e 123 não-portadoras. As variáveis contínuas foram analisadas pelo teste $t$ de Student, e as categóricas pelos testes do $\chi^{2}$ e exato de Fisher, por meio do software Epi-Info 2000 (CDC, Atlanta). RESULTADOS: ocorreu morbidade puerperal em 18 pacientes do grupo HIV-casos (22\%) e 17 do grupo-controle (14\%), com predomínio das variáveis de morbidade menor, sem diferença significativa entre os grupos, exceto pelo risco mais alto de endometrite no grupo HIV-casos (RR=1,05; IC a 95\%: 1, 0 1-1, 1). Não foi observada diferença significativa entre os grupos quanto aos tipos de parto. Houve somente duas ocorrências de morbidade maior: hemotransfusão e fasciite necrotizante. CONCLUSÕES: puérperas portadoras do HIV apresentam morbidade semelhante à das puérperas não-portadoras do vírus, apesar da predominância de morbidade menor e do risco aumentado de endometrite no grupo portador do vírus. $\bigcirc$ acompanhamento clínico no puerpério imediato é estratégico para a identificação precoce da morbidade materna.

\section{Abstract}

PURPOSE: to evaluate puerperal morbidity in HIV-infected and HIV non-infected puerperal women. METHODS: longitudinal and controlled study performed from July 2001 to September 2003, in 205 pregnant women admitted for birth delivery at Odete Valadares Maternity, divided in two groups: HIV-infected women (82) and HIV non-infected women (123). Postpartum morbidity evaluation was performed from birth delivery up to 15 days postpartum. Morbidity was categorized as minor (postpartum hemorrhage, fever and endometritis) or major (blood transfusion, deep alterations of the surgical wound and indication for surgical intervention), and was evaluated both according to the presence or absence of HIV infection and the mode of delivery. Continuous variables were analyzed by the Student's ttest, and categorical variables were analyzed by $\chi^{2}$ and Fisher's exact test using Epi-Info 2000 (CDC, Atlanta). RESULTS: puerperal morbidity was observed in 18 patients from the HIV group (22\%) and in 17 patients from the control group (14\%) with predominance of minor morbidity, without statistical significance, except for an increased risk of endometritis in the HIV group (RR= 1.05; Cl 95\%:1.01-1 . 10). No significant difference was observed concerning the mode of delivery between the two groups. There were only two major morbidities: blood transfusion and necrotizing fasciitis. CONCLUSIONS: HIV-infected and non-infected puerperal women have a similar morbidity, despite the lower morbidity in the HIV non-infected group and the increased risk of endometritis in the HIV group. Clinical puerperium follow-up is a strategic control tool for an early identification of maternal morbidity.
Maternidade Odete Valadares, Fundação Hospitalar do Estado de Minas Gerais - FHEMIG - Belo Horizonte (MG), Brasil.

' Chefe da Divisão Assistencial da Maternidade Odete Valadares - Fundação Hospitalar do Estado de Minas Gerais - FHEMIG - Belo Horizonte (MG), Brasil.

2 Professores Adjuntos da Faculdade de Medicina da Universidade Federal de Minas Gerais - UFMG - Belo Horizonte (MG), Brasil.

${ }^{3}$ Médica do Serviço de Obstetrícia da Maternidade Odete Valadares - Fundação Hospitalar do Estado de Minas Gerais - FHEMIG

- Belo Horizonte (MG), Brasil. 


\section{Introdução}

Intervenções clínicas com o uso ampliado da terapia antiretroviral têm reduzido de forma significativa a transmissão vertical do vírus da imunodeficiência humana (HIV), baseado no controle da carga viral plasmática materna ${ }^{1}$.

Estudos observacionais, randomizados e de metanálise mostram que a cesariana realizada antes da ruptura das membranas e/ou do trabalho de parto é fator protetor aditivo à terapia anti-retroviral em gestantes com carga viral plasmática superior a 1.000 cópias $/ \mathrm{mL}^{2,3}$.

Entretanto, a segurança materna dos procedimentos operatórios deve ser avaliada e estabelecida. São consideradas situações de risco a presença de graus mais avançados de imunodeficiência, ausência de uso de medicação anti-retroviral e cesarianas realizadas após trabalho de parto e/ou ruptura das membranas ${ }^{4-7}$.

Os resultados de estudos retrospectivos e prospectivos indicam aumento da morbidade materna associada ao parto e, principalmente, à cesariana em mulheres portadoras do HIV, quando comparadas a mulheres não-portadoras. A análise de 1.186 partos na maior coorte puerperal realizada na América do Norte demonstrou morbidade materna de $15 \%$. A cesariana eletiva foi fator de risco independente de morbidade pós-parto em geral e, especificamente, no caso de febre sem infecção. A cesariana intraparto foi fator de risco de endometrite ${ }^{8}$.

Marcollet et al. ${ }^{9}$ conduziram pesquisa comparativa entre as diferentes vias de parto em 401 pacientes portadoras do HIV. Entre estas, 200 foram submetidas a parto vaginal e 201 à cesariana (92 por emergência e 109 eletivas). Uma ou mais complicações ocorreram em $12 \%$ das cesarianas de emergência, em $6,4 \%$ das cesarianas eletivas e em $4 \%$ dos partos vaginais $(\mathrm{p}=0,04)$. $\mathrm{Na}$ análise multivariada ajustada por níveis de CD4 + e hemorragia anteparto, o risco relativo de qualquer complicação pós-parto (maior ou menor) foi 1,85 (IC 95\%:1,0-3,4) após cesariana eletiva e 4,17 (IC a 95\%:2,3-7,5) após cesariana de emergência, quando comparadas ao parto vaginal $(\mathrm{p}=0001)$.

Os resultados da coorte suíça, em estudo multicêntrico controlado, mostraram aumento da incidência de morbidade menor e anemia pós-parto, independentemente do controle de carga viral em pacientes submetidas à cesariana eletiva ${ }^{10}$.

Estudo observacional não controlado com coorte perinatal de gestantes infectadas pelo HIV residentes na América Latina e Caribe, com o objetivo de analisar a morbidade puerperal, incluiu 697 gestantes, das quais
470 eram brasileiras. Ocorreram 299 partos vaginais, 260 cesarianas eletivas e 138 cesarianas intraparto. Foi encontrada morbidade pós-parto em $36(5 \%)$ mulheres (18 leves e 18 graves), com associação significante para a cesariana $(\mathrm{p}=0,02)$. Houve aumento de morbidade menor em pacientes portadoras do HIV submetidas à cesariana intraparto $(\mathrm{OR}=2,96)$ e após ruptura das membranas $(\mathrm{OR}=1,16)^{11}$.

As complicações mais freqüentes relatadas na literatura para estas mulheres são endometrite, infecção da incisão cirúrgica, infecção do trato urinário, anemia, necessidade de hemotransfusão e pneumonia, além de mais tempo de internação hospitalar ${ }^{4,6,7,12,13}$. É relatado o aumento da morbidade materna em gestantes soropositivas para o HIV submetidas à cesariana. Essas pacientes apresentaram mais incidência de febre, endometrite e infecção urinária ${ }^{4}$. Em estudo caso-controle comparando 45 gestantes e 90 controles emparelhadas pela indicação de cesariana e data do parto, observou-se taxa de complicações pós-cesariana de $86,7 \%$ - sendo mais freqüentes anemia, febre e infecção urinária - e mais tempo de internação hospitalar quando comparado aos controles ${ }^{12}$.

Um estudo prospectivo incluiu 307 mulheres pareadas por idade materna e peso, submetidas à cesariana, das quais $88 \%$ foram de emergência. Entre o grupo total de pacientes, 248 eram soronegativas para o HIV, enquanto 59 tinham a sorologia positiva. A endometrite foi mais comum entre as pacientes portadoras do HIV e relacionada com níveis de linfócitos T CD4 + abaixo de 500 células $/ \mathrm{mm}^{313}$.

Entretanto, ainda existem poucas avaliações sobre a morbidade puerperal na população brasileira e os resultados são divergentes dos de outras populações. O único estudo controlado, apesar de retrospectivo, com 58 gestantes brasileiras portadoras do HIV, emparelhadas a 158 controles por idade materna e índice de massa corporal (IMC), não mostrou aumento da morbidade puerperal nas mulheres infectadas. Em relação à metodologia, a seleção do grupo-controle foi feita por meio da sorologia determinada no prénatal, em diferentes idades gestacionais, não havendo informações da sorologia no momento do parto ${ }^{14}$. A coorte perinatal prospectiva envolveu muitas mulheres brasileiras, mas foi um estudo observacional não controlado, tendo avaliado somente a morbidade em puérperas portadoras do HIV, com seguimento entre seis e 12 semanas após o parto, e seis meses depois do parto ${ }^{11}$.

Assim, não existe estudo controlado brasileiro que avalie as primeiras semanas do puerpério das gestantes infectadas pelo HIV. O objetivo deste trabalho é, 
portanto, avaliar a morbidade imediata de puérperas portadoras do HIV, comparadas a puérperas não-portadoras do vírus.

\section{Métodos}

Estudo observacional controlado para descrever a morbidade relacionada com a via de parto em pacientes portadoras do HIV. As pacientes foram admitidas para o parto na Maternidade Odete Valadares da Fundação Hospitalar do Estado de Minas Gerais (FHEMIG), integrantes da rede assistencial do Sistema Único de Saúde (SUS) da região metropolitana de Belo Horizonte, MG.

Elas foram selecionadas no período entre julho de 2001 e setembro de 2003, e comparadas a pacientes não-portadoras do vírus. Definiu-se como grupo HIV-casos as pacientes portadoras do HIV e grupo controle (controles) as não-portadoras selecionadas no puerpério imediato.

Os critérios de emparelhamento para formação do grupo controle foram teste rápido anti-HIV negativo no parto, tipo de parto, idade gestacional no parto, idade materna e parto no mesmo período do caso (24 horas até 30 dias do caso). Esse período de parto entre casos e controles seguiu os mesmos parâmetros utilizados pela Comissão de Controle de Infecções Hospitalares (CCIH) da Maternidade Odete Valadares para investigação de morbidade puerperal infecciosa.

Os critérios de inclusão para o grupo HIV-casos foram: parto realizado na instituição; ausência de doença crônica prévia (hipertensão, diabetes mellitus, doença pulmonar crônica, tromboembolismo, doença auto-imune) e/ou intercorrências da gestação (diabetes gestacional, pré-eclâmpsia, pielonefrite aguda). O diagnóstico de infecção pelo HIV e/ou AIDS seguiu o protocolo padronizado pela Coordenação Nacional de DST/AIDS do Ministério da Saúde. Para o diagnóstico do estágio da doença foi utilizada a classificação do Centers for Prevention and Diseases Control (CDC), publicada em 1993. Essa classificação apresenta nove categorias, levando em consideração a contagem de linfócitos T CD4 + em três diferentes níveis, associada à condição clínica do paciente portador do HIV estratificada em três classes, de acordo com a progressão para AIDS. No nível mais inicial (classe A1), o paciente é portador assintomático, apresentando-se com imunidade íntegra (contagem de células T CD 4 + acima de 500 células $/ \mathrm{mm}^{3}$ ). No grau mais avançado da doença, o paciente apresenta-se com sintomatologia (ou é portador de alguma doença indicadora de AIDS) e encontra-se imunossuprimido, apresentando contagem de células T CD $4+$ abaixo de 200 células $/ \mathrm{mm}^{3}$ (classe C3).

Os critérios de inclusão para o grupo controle foram: parto realizado na instituição; ausência de infecção pelo HIV e/ou AIDS, conforme critérios estabelecidos pelo CDC em 1993; ausência de doença crônica prévia (hipertensão, diabetes mellitus, doença pulmonar crônica, tromboembolismo, doença autoimune) e/ou intercorrências da gestação (diabetes gestacional, pré-eclâmpsia, pielonefrite aguda). Para rastreamento de infecção no grupo-controle, foi utilizado o teste ELISA rápido (Determine ${ }^{\circledR}$, Abott Inc.), padronizado pela Coordenação Nacional de DST/AIDS do Ministério da Saúde e recomendado para o uso em parturientes em situações de emergência. O teste foi realizado após consentimento, e todas as pacientes foram informadas do resultado, sendo aconselhadas conforme o protocolo assistencial da instituição em acordo com as normas instituídas pelo Ministério da Saúde para testagem durante o parto.

Os critérios de exclusão adotados foram: determinação da sorologia anti-HIV por outro método ou teste não padronizado no estudo.

As pacientes foram acompanhadas em dois períodos: no pós-parto imediato até a alta hospitalar; após a alta eram agendadas para uma visita ambulatorial entre o sétimo e o $15^{\circ}$ dia de puerpério. Caso houvesse indicação de internação hospitalar pós-alta, eram acompanhadas pelos pesquisadores. Os dados foram coletados em questionários específicos para cada período-visita.

Baseado em dados observacionais da instituição e considerando-se taxa de morbidade de $20 \%$ nos casos e $5 \%$ nos controles, foi calculado tamanho amostral de 60 pacientes por grupo (intervalo de confiança de $95 \%$ e poder de detecção de $80 \%$ ).

Os tipos de parto considerados foram: cesariana eletiva, ou seja, realizada antes do início do trabalho de parto e com membrana amniótica íntegra; cesariana de emergência, realizada após início do trabalho de parto e/ou ruptura de membranas amnióticas; parto vaginal sem manobras de extração fetal ou uso de fórceps e/ou vácuo-extrator.

As variáveis de morbidade materna foram divididas em duas categorias previamente estabelecidas e baseadas em estudos publicados até o ano de 2001.

Considerou-se morbidade menor: febre sem sinais e/ou sintomas de processo infeccioso (duas medidas de temperatura axilar iguais ou acima de $38^{\circ} \mathrm{C}$, após 24 horas de pós-parto, excluídas as alterações clínicas e laboratoriais descritas a seguir e alterações da lactação); endometrite (morbidade febril associada à 
involução uterina incompatível com a idade puerperal e/ou lóquios com odor alterado e/ou dor à mobilização uterina no exame pélvico); infecção do trato urinário (alteração do sedimento urinário - nitrito positivo e/ou número superior a 10 piócitos/campo associado à presença de bactérias coráveis pelo método de Gram e/ou urocultura positiva - acima de 10.000 unidades formadoras de colônia); alterações da ferida operatória (seroma, celulite, abscesso e/ou hematoma, definidos clinicamente e confinados ao tecido celular subcutâneo); aumento do sangramento pós-parto (definido pela observação clínica e/ou necessidade de, pelo menos, uma das seguintes intervenções: uso de fármacos uterotônicos, necessidade de revisão de cavidade uterina e canal de parto, troca de curativo da incisão cirúrgica e curetagem pós-parto).

Considerou-se morbidade maior: necessidade de intervenção cirúrgica após o parto (laparotomia exploradora, drenagem de abscesso pélvico, histerectomia, drenagem de hematoma subaponeurótico e/ou pélvico, fasciite necrotizante); necessidade de hemotransfusão; critérios definidores de síndrome de resposta inflamatória sistêmica e/ou sepsis (taquicardia, taquipnéia, hiper ou hipotermia associadas ou não à hipotensão); pneumonia; morbidade febril associada a alterações de ausculta respiratória e/ou da radiografia de tórax; tromboembolismo (alterações respiratórias e/ou edema assimétrico de membros inferiores confirmados por cintilografia pulmonar e/ou duplex-scan venoso de membros inferiores), conforme Quadro 1.

Para a construção do banco de dados, foram utilizados os programas Epi Info 2002 - versão 2 (CDC, Atlanta 2002), Excel 8.0 (Microsoft Inc., 1997) e SPSS 8.0 for Windows (SPSS Inc., 1997). As variáveis contínuas (idade materna, idade gestacional no parto e contagem de linfócitos T CD4+) foram analisadas pelo teste $t$ de Student e pelo Kruskal-Wallis. As variáveis dicotômicas (presença ou ausência de morbidade, presença ou ausência de critérios de morbidade maior e menor) foram avaliadas pelos testes do $\chi^{2}$ com correção de Yates ou o teste exato de Fisher. Considerou-se nível de significância de $95 \%$. O risco foi estimado com base no cálculo do risco relativo.
O trabalho foi aprovado pela Comissão de Ética da Maternidade Odete Valadares da FHEMIG (parecer emitido em março de 2001). Todas as pacientes assinaram o Termo de Consentimento Livre e Esclarecido.

Foram incluídas no estudo 205 puérperas, no período de julho de 2001 a setembro de 2003 . As pacientes foram acompanhadas no período de internação, no parto e no período puerperal até 15 dias após o parto. Entre as 205 puérperas, 82 eram portadoras do HIV (grupo HIV-casos) e 123 apresentaram sorologia negativa no parto (grupo controle), com proporção entre casos e controles de 1:1,5.

No grupo HIV-casos, as 80 puérperas estavam em uso de antiretrovirais: 25 em monoterapia com zidovudina; 27 em terapia tríplice com inibidor de protease; 20 em terapia tríplice com nevirapina e oito com outros esquemas de terapia.

Todas as pacientes incluídas no estudo (grupo HIV-casos e grupo controle) e que foram submetidas à cesariana eletiva ou de emergência receberam uma dose de cefalotina ( $1 \mathrm{~g}$ endovenoso após clampagem do cordão umbilical), conforme o protocolo de antibioticoprofilaxia da Comissão de Controle de Infecção Hospitalar da Maternidade Odete Valadares.

\section{Resultados}

Os grupos foram homogêneos quanto à idade materna e idade gestacional no parto. A média de idade materna foi de 27,8 e 27,1 anos, respectivamente, nos grupos HIV-casos e controle. A média de idade gestacional foi de 37,8 no grupo HIV-casos e 38,1 semanas no grupo controle, sem diferença significativa.

Em 48 pacientes do grupo HIV-casos foi possível determinar os níveis de linfócitos T CD4+, no período de até 30 dias antes do parto. A média de contagem de linfócitos T CD $4+$ foi de 431 células $/ \mathrm{mm}^{3}$, sendo que seis pacientes apresentaram níveis inferiores a 200 células $/ \mathrm{mm}^{3}$. Nenhuma apresentou sinais e sintomas definidores de AIDS durante o período do estudo.

Ocorreu morbidade puerperal em 18 integrantes do grupo HIV-casos (22\%) e em 17 do grupo controle

Quadro 1 - Variáveis de morbidade materna distribuídas por graus de gravidade.

\begin{tabular}{|ll|}
\hline Morbidade maior & Morbidade Menor \\
\hline Necessidade de intervenção cirúrgica após o parto & Febre \\
Necessidade de hemotransfusão & Endometrite \\
Pneumonia & Infecç̃o do trato urinário \\
Trombose venosa profunda e tromboembolismo pulmonar & Alteração subcutânea da incisão cirúrgica \\
Sinais clínicos de síndrome de resposta inflamatória sistêmica & Aumento de sangramento pós-parto sem necessidade de reposição volêmica e/ou hemotransfusão \\
\hline
\end{tabular}

Traduzido e adaptado de Semprini et al. (1995) 4 . 
(14\%), sem diferença significativa, conforme demonstrado na Tabela 1.

Não foi observado aumento significativo da morbidade relacionada ao tipo de parto entre os dois grupos (Tabela 2).

Em relação à categoria de morbidade (maior ou menor), houve predominância de variáveis de morbidade menor. Entre estas, no grupo HIV-casos, a febre foi o achado mais freqüente $(12,2 \%)$, seguida das alterações subcutâneas da ferida operatória (11\%), endometrite $(4,9 \%)$ e sangramento aumentado $(2,4 \%)$. Apenas uma paciente do grupo HIV-casos necessitou de hemotransfusão e uma do grupo controle apresentou quadro clínico de fasciite necrotizante após parto vaginal.

$\mathrm{Na}$ análise estratificada por variável de morbidade menor nos dois grupos, foi observado resultado estatisticamente significativo somente em relação à endometrite $(\mathrm{RR}=1,05 ; \mathrm{IC}$ a 95\%:1,01-1,10; $\mathrm{p}=0,02)$, segundo a Tabela 3. Entretanto, não houve diferença quando se analisou a relação entre endometrite e tipo de parto: $4,3 \%$ das pacientes submetidas à cesariana de emergência $(\mathrm{p}=0,39)$; $4,1 \%$ das submetidas à cesariana eletiva $(\mathrm{p}=0,16) ; 2,8 \%$ das submetidas a parto vaginal $(\mathrm{p}=0,39)$. Não ocorreu endometrite entre as pacientes do grupo controle.

Nos 48 casos do grupo HIV-casos em que foi determinada a contagem de linfócitos T CD4+, observou-se

Tabela 1 - Distribuição da frequiência de morbidade puerperal nos grupos do estudo.

\begin{tabular}{l|ccccccc}
\hline & \multicolumn{2}{c}{$\begin{array}{c}\text { Grupo HIV- } \\
\text { casos }\end{array}$} & \multicolumn{2}{c}{$\begin{array}{c}\text { Grupo } \\
\text { controle }\end{array}$} & RR (IC a 95\%) & \multirow{2}{*}{ p $^{*}$} \\
\cline { 2 - 5 } & $\mathrm{N}$ & $\%$ & \multicolumn{1}{c}{$\mathrm{N}$} & $\%$ & & \\
\hline Morbidade & 18 & 22 & 17 & 14 & $1,10(0,96-1,2)$ & 0,13 \\
Sem morbidade & 64 & 78 & 106 & 86 & & \\
Total & 82 & & 123 & & & \\
\hline
\end{tabular}

Teste do $\chi^{2}$. morbidade em oito, sendo dois (endometrite e febre) em pacientes com contagem de células CD4+ abaixo de 200 células $/ \mathrm{mm}^{3}$.

Não foi observada diferença significativa da incidência de morbidade menor entre as pacientes do grupo HIV-casos em uso de monoterapia com zidovudina $(16 \%)$ e de outros esquemas antiretrovirais múltiplos $(23 \% ; \mathrm{p}=0,99)$.

Em relação ao período de observação clínica, metade dos casos de morbidade no grupo HIV-casos e todos os casos de endometrite puerperal foram diagnosticados na visita ambulatorial ou na reinternação hospitalar no período até 15 dias após o parto.

\section{Discussão}

A incidência de morbidade puerperal em portadoras do HIV é variável, dependendo do desenho do estudo, tipo de parto e características clínicas e imunológicas do grupo (5 a 86\%). Existe risco mais alto diante de graus mais avançados de imunodeficiência, ausência de uso de medicação antiretroviral e cesarianas realizadas após trabalho de parto e/ou ruptura das membranas ${ }^{11,14-16}$.

Estudos controlados e não-controlados observaram ausência de aumento do risco de morbidade materna em pacientes portadoras do HIV submetidas à cesariana eletiva, quando comparadas a mulheres não-portadoras $^{7,17,18}$. Entretanto, em coorte controlada avaliando risco de morbidade em pacientes soropositivas para o HIV com adequado controle da carga viral, verificou-se risco elevado de morbidade menor quando comparadas a não-portadoras do vírus (febre, anemia e infecção do trato urinário $)^{10}$.

Tabela 2 - Distribuição dos casos de morbidade puerperal por tipo de parto e grupo do estudo.

\begin{tabular}{lcccc}
\hline Tipo de parto & Grupo HIV-casos & Grupo controle & Total \\
n/N & n/N & 119 & RR (IC a 95\%) \\
\hline Cesariana eletiva & $12 / 48$ & $13 / 71$ & 28 & $1,08(0,89-1,3) \#$ \\
Cesariana intraparto & $3 / 11$ & $3 / 17$ & 58 & $1,13(0,7-1,4)^{*}$ \\
Parto vaginal & $3 / 23$ & $1 / 35$ & 205 & $4,57(0,5-41,2)^{*}$ \\
Total & $18 / 82$ & $17 / 123$ & - \\
\hline
\end{tabular}

*Teste exato de Fisher; \# teste do $\chi^{2}$

Tabela 3 - Distribuição das variáveis de morbidade menor nos grupos HIV e controle.

\begin{tabular}{|c|c|c|c|c|}
\hline Morbidade Menor & $\begin{array}{c}\text { Grupo HIV-casos } \\
\text { n/N }\end{array}$ & $\begin{array}{c}\text { Grupo controle } \\
\text { n/N }\end{array}$ & $\begin{array}{c}\text { Total } \\
\mathbf{N}\end{array}$ & RR (IC a 95\%) \\
\hline Febre & $10 / 82$ & $10 / 123$ & 205 & $1,04(0,95-1,15)$ \\
\hline Alteração da ferida operatória & $9 / 82$ & $9 / 123$ & 205 & $1,04(0,95-1,14)$ \\
\hline Sangramento & $2 / 82$ & $0 / 123$ & 205 & $1,02(0,99-1,06)$ \\
\hline ITU & $1 / 82$ & $1 / 123$ & 205 & $1,0 \quad(0,1-1,03)$ \\
\hline Endometrite & $4 / 82$ & $0 / 123$ & 205 & $1,05(1,01-1,10)^{\star}$ \\
\hline Total & $26 / 82$ & $20 / 123$ & 205 & \\
\hline
\end{tabular}

* $\mathrm{p}=0,02$, Teste exato de Fisher; ITU: infecção do trato urinário. 
Os critérios adotados para a seleção das pacientes neste estudo foram semelhantes aos de Semprini et al. ${ }^{4}$, quanto à proximidade do parto, e ao de Grubert et al. ${ }^{5}$, quanto à idade materna e gestacional. Ao contrário de outros trabalhos, foram excluídas da presente investigação as pacientes com história de doenças crônicas prévias à gestação (diabetes, hipertensão arterial, pneumopatias, alterações auto-imunes e anemias) e intercorrências clínicas na gestação atual (pré-eclâmpsia, diabetes gestacional, pielonefrite).

Os critérios de definição de morbidade adotados foram baseados em outros trabalhos sobre o tema ${ }^{4,13,15}$.

Escolheu-se um desenho prospectivo semelhante ao já adotado ${ }^{13,17}$. Entretanto, nessas pesquisas, o seguimento das puérperas foi limitado ao período da internação hospitalar e parto, enquanto na presente, o acompanhamento estendeu-se até 15 dias após o parto.

Watts et al. ${ }^{7}$, acompanhando 497 puérperas infectadas pelo HIV (coorte não-controlada), encontraram freqüência significativamente maior de endometrite na análise multivariada, de forma decrescente, de acordo com o tipo de parto: $27 \%$ para as cesarianas de emergência (intraparto); $16 \%$ para as cesarianas eletivas; $7 \%$ para o parto vaginal. No nosso estudo, a freqüência de endometrite no grupo HIV-casos, de acordo com o tipo de parto, foi bem menor e não houve diferença significante entre os diferentes modos de parto: $4,3 \%$ para as pacientes submetidas à cesariana de emergência; $4,1 \%$ para as cesarianas eletivas; $2,8 \%$ para as pacientes com parto vaginal.

Outro aspecto a ser analisado foi a determinação da sorologia anti-HIV do grupo controle. Nesta investigação, optou-se pela determinação da sorologia anti-HIV a partir da testagem rápida pelo método ELISA imunocromatográfico, independentemente do resultado da sorologia realizada na assistência pré-natal, o que permitiu testagem sorológica por metodologia padronizada de alta especificidade. O teste foi efetuado dentro do período de acompanhamento das puérperas, minimizando a possibilidade de inclusão de pacientes portadoras do HIV no grupo controle. Em outros estudos, no entanto, a seleção dos controles foi baseada na sorologia pré-natal ${ }^{13,14}$.

A freqüência de morbidade materna encontrada nas portadoras de HIV foi de $22 \%$, com predominância de morbidade menor (febre, endometrite e alterações subcutâneas da ferida operatória), sem diferença significativa quando comparada a puérperas não-portadoras. $\mathrm{O}$ estudo colaborativo europeu, analisando 408 puérperas portadoras do HIV comparadas a 408 não-portadoras, demonstrou freqüência de 29,2\% de morbidade, com predominância de morbidade menor em pacientes submetidas a parto vaginal (febre) e cesariana eletiva (anemia sem necessidade de hemotransfusão) ${ }^{19}$.
$\mathrm{Na}$ análise estratificada das variáveis de morbidade menor, verificou-se risco mais alto de endometrite nas pacientes portadoras do vírus. Apesar disso, a freqüência de endometrite $(4,8 \%)$ foi menor que a relatada no estudo prospectivo controlado sul-africano, que avaliou a morbidade imediata $(23 \%)^{13}$. A maior freqüência de endometrite pode estar associada ao uso da antibioticoprofilaxia intraparto na totalidade das pacientes submetidas à cesariana, em concordância com recente pesquisa comparativa, não-controlada, envolvendo gestantes tailandesas, e com estudo colaborativo europeu, com uso de antibioticoprofilaxia em todas as pacientes, apresentando freqüência de endometrite de 2,2 e 1,2\%, respectivamente ${ }^{18,19}$.

Esta casuística não teve poder suficiente para demonstrar aumento do risco de morbidade em cesarianas de emergência nas pacientes infectadas pelo HIV, quando comparadas ao parto vaginal e cesariana eletiva, embora este fato não possa ser afastado, em concordância com os resultados da coorte perinatal da América Latina e Caribe e do estudo colaborativo europeu $^{11,19}$. A freqüência de morbidade na coorte perinatal foi de 5\%, com predominância de morbidade menor, associada ao modo de parto, apresentando mais risco, quando associado à cesariana intraparto e/ou rotura das membranas. No estudo colaborativo europeu, o risco de morbidade menor foi mais elevado nas mulheres portadoras do HIV submetidas à cesariana eletiva, quando comparadas às submetidas ao parto vaginal $\left(\mathrm{RR}=2,3\right.$; IC a 95\%:1,8-2,9) ${ }^{11,19}$.

$\mathrm{O}$ uso de antiretrovirais para o tratamento da infecção pelo HIV e a contagem de linfócitos T CD4+ abaixo de 200 células $/ \mathrm{mm}^{3}$ estão associados à morbidade puerperal materna ${ }^{6,11}$. Esta pesquisa não teve poder para demonstrar essa associação, devido ao número limitado de pacientes com contagem de linfócitos T CD4+ até 30 dias da data do parto e de pacientes em uso de antiretrovirais para tratamento da infecção pelo HIV.

Um dado clínico de relevância foi a identificação de metade dos casos de morbidade e de todos os casos de endometrite após a alta hospitalar. Este fato reforça a necessidade do seguimento clínico em curto prazo dessas puérperas.

Concluindo, as puérperas portadoras do HIV apresentam morbidade puerperal semelhante a puérperas não-portadoras, com predominância de morbidade menor e com risco aumentado de endometrite. A redução da incidência de morbidade nessas mulheres deve ser avaliada em estudos que considerem o grau de imunodeficiência, uso de antibioticoprofilaxia no parto e de antiretrovirais indicados para tratamento da infecção pelo HIV. 
1. loannidis JPA, Abrams EJ, Ammann A, Bulterys M, Goedert JJ, Gray L, et al. Perinatal transmission of human immunodeficiency virus type 1 by pregnant women with RNA viral loads $<1.000$ copies/ml. J Infec Dis. 2001;183(4):539-45.

2. European Mode of Delivery Collaboration. Elective caesarean-section versus vaginal delivery in prevention of vertical HIV-1 transmission: a randomised clinical trial. Lancet. 1999;353(9158):1035-9.

3. International Perinatal HIV Group. The mode of delivery and the risk of vertical transmission of human immunodeficiency virus type 1: a meta-analysis of 15 prospective cohort studies. N Engl J Med. 1999;340(13):977-87.

4. Semprini AE, Castagna C, Ravizza M, Fiore S, Savasi V, Muggiasca $\mathrm{ML}$, et al. The incidence of complications after caesarean section in 156 HIV-positive women. AIDS. 1995;9(8):913-7.

5. Grubert TA, Reindell D, Kastner R, Lutz-Friedrich R, Belohradsky $\mathrm{BH}$, Dathe $\mathrm{O}$. Complications after caesarean section in HIV1 -infected women not taking antiretroviral treatment. Lancet. 1999;354(9190):1612-3.

6. Bjorklund K, Mutyaba T, Nabunya E, Mirembe F. Incidence of postcesarean infections in relation to HIV status in a setting with limited resources. Acta Obstet Gynecol Scand. 2005;84(10):967-71.

7. Watts DH, Lambert JS, Stiehm ER, Bethel J, Whitehouse J, Fowler MG, et al. Complications according to mode of delivery among human immunodeficiency virus-infected women with CD4 lymphocyte counts of $<$ or $=500 /$ microl. Am J Obstet Gynecol. 2000; 183(1):100-7.

8. Read JS, Tuomala R, Kpamegan E, Zorrilla C, Landesman S, Brown $G$, et al. Mode of delivery and postpartum morbidity among HIVinfected women: the women and infants transmission study (WITS). J Acquir Immun Def Syndr. 2001 ;26(3):236-45.

9. Marcollet A, Goffinet F, Firtion G, Pannier E, Le Bret T, Brival ML, et al. Differences in postpartum morbidity in women who are infected with the human immunodeficiency virus after elective cesarean delivery, emergency cesarean delivery, or vaginal delivery. Am J Obstet Gynecol. 2002; 186(4):784-9.

10. Lapaire $O$, Irion $O$, Koch-Holch A, Holzgreve W, Rudin C, Hoesli I, et al. Increased peri- and post-elective cesarean section morbidity in women infected with human immunodeficiency virus-1: a case-controlled multicenter study. Arch Gynecol Obstet. 2006;274(3):165-9.

11. Duarte G, Read JS, Gonin R, Freimanis L, Ivalo S, Melo VH, et al. Mode of delivery and postpartum morbidity in Latin American and Caribbean countries among women who are infected with human immunodeficiency virus-1: the NICHD International Site Development Initiative (NISDI) Perinatal Study. Am J Obstet Gynecol. 2006; 195(1):215-29.

12. Maiques-Montesinos V, Cervera-Sanchez J, Bellver-Pradas J, Abad-Carrascosa A, Serra-Serra V. Post-cesarean section morbidity in HIV-positive women. Acta Obstet Gynecol Scand. 1999;78(9):789-92.

13. Urbani G, de Vries MM, Cronje HS, Niemand I, Bam RH, Beyer $\mathrm{E}$, et al. Complications associated with cesarean section in HIVinfected patients. Int J Gynaecol Obstet. $2001 ; 74(1): 9-15$.

14. Rocco R, Leite HV, Vasconcellos M, Cabral ACV. Morbidade associada a cesariana eletiva em portadoras do HIV. Rev Bras Ginecol Obstet. 2003;25(5):323-8.

15. Rodriguez EJ, Spann C, Jamieson D, Lindsay M. Postoperative morbidity associated with cesarean delivery among human immunodeficiency virus-seropositive women. Am J Obstet Gynecol. 2001;184(6):1108-11.

16. Ferrero S, Bentivoglio G. Post-operative complications after caesarean section in HIV-infected women. Arch Gynecol Obstet. $2003 ; 268(4): 268-73$

17. Vimercati A, Greco P, Loverro G, Lopalco PL, Pansini V, Selvaggi L. Maternal complications after caesarean section in HIV infected women. Eur J Obstet Gynecol Reprod Biol. 2000;90(1):73-6.

18. Panburana $P$, Phaupradit $W$, Tantisirin $O$, Sriintravanit $N$, Buamuenvai J. Maternal complications after caesarean section in HIV-infected pregnant women. Aust N Z J Obstet Gynaecol. 2003;43(2): 160-3.

19. Fiore S, Newell ML, Thorne C, European HIV in Obstetrics Group. Higher rates of post-partum complications in HIV-infected than in uninfected women irrespective of mode of delivery. AIDS. 2004; 18(6):933-8. 